\title{
The Application of QR Codes And WIFI Technology in the Autonomous Navigation System for AGV
}

\author{
Chuanhong Zhou ${ }^{1, a}$, Pujia Shuai ${ }^{1, b}$ and Chao Dai ${ }^{1, c}$ \\ ${ }^{1}$ Department of School of Mechatronic Engineering and Automation, Shanghai University, Shanghai \\ 200072, P.R.China. \\ a13817297415@139.com, b512100799@qq.com, c2450530628@qq.com
}

Keywords: QR code, AGV, path planning, positioning information

Abstract. With the development of Internet, the two-dimensional code technology has been widely used. It is a technology which includes information coding, information transmission, images processing and data encryption. And it develops from one-dimension bar code. It can realize rapid positioning and automatic navigation because of the functions above. The static path planning and dynamic path planning are adapted when the map of AGV is built. At the same time, this system also adapts the WIFI positioning technology to avoid missing the two-dimensional code.

\section{Introduction}

AGV (automatic guided vehicle) applied in industry can achieve the goal of unmanned and work through computers. Aimed at the problem of positioning accuracy and costs mentioned, this paper will introduce QR codes technology[1]. How AGV to achieve rapid positioning?

(1) First, post the two-dimensional code label on the ground. And the positioning information is stored in the label.

(2) Second, when the AGV passes the area, it will scan and analysis the two-dimensional code through the camera comes with AGV.

QR code (abbreviated from Quick Response Code) is the trademark for a type of matrix barcode. The basic structure of QR codes[2] is shown as Fig. 1:

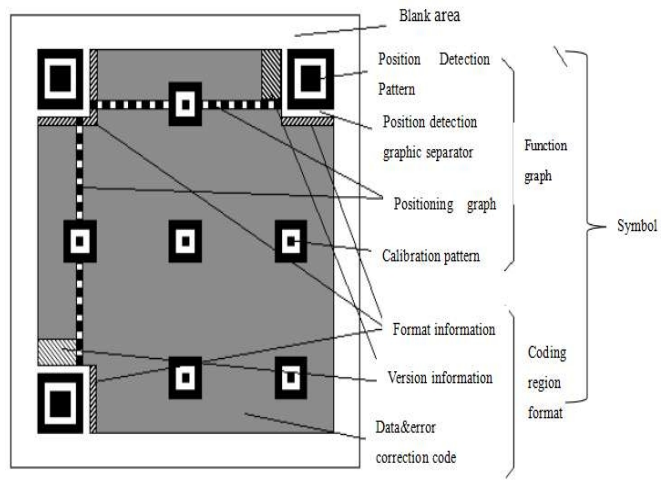

Fig. 1. The basic structure of QR codes

The position detection pattern is a positioning pattern used to mark the rectangular size of a two-dimensional code. The white edges of the three positioning patterns are called position probe pattern separators. These three position detection patterns form a square with the non-probing pattern in the lower right corner. The order from top left to top right is: $0,1,2,3$.

However, when AGV is driving, there exists some positioning deviation because of some uncontrollable factors. So, this system also adapts WIFI positioning technology.

\section{Positioning design}

The design idea of this paper is that we use the scanning module to scan the two-dimensional code coordinate information posted on the ground and then send the information processed by a certain algorithm to AGV control system[3]. Finally we will use the control module to guide the next 
operation of AGV. The operation needs to read the information of two-dimensional code and measure the distance from AGV to the code, as well as obtain the position determination of AGV. The specific flow chart is shown as Fig. 2:

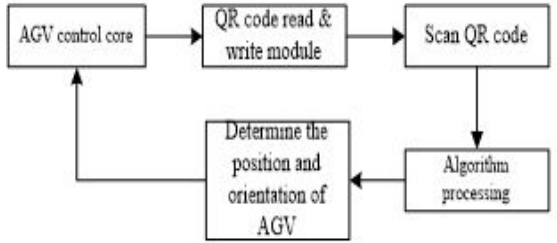

Fig. 2. The flow chart of AGV's positioning scheme

The control system of $\mathrm{AGV}$ is divided into three parts which are upper control management scheduling system[4], local control system of AGV and navigation system. The relationship between them is shown as Fig. 3:

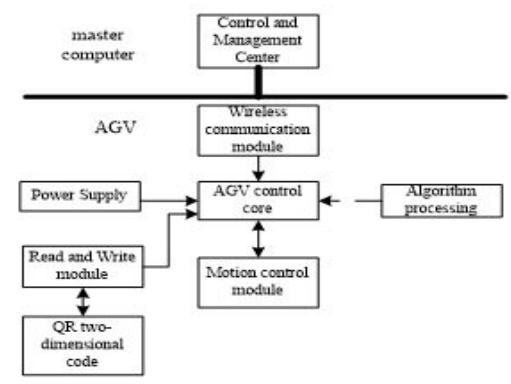

Fig. 3. System block diagram

In the above figure, the core control modules mainly include two parts which are motion control module and the algorithm processing of two-dimensional code. They are responsible for processing of the scanned result and controlling the movement of AGV. The reader/writer module of two-dimensional code is used to obtain the positioning information of the QR code labels which are posted on the ground according to the arrangement of the ranks. QR codes are widely used in our country, so we generate the two-dimensional code according to QR code standard.

The acquisition of the two-dimensional code's information. In order to ensure the organic integration of generation module and system, this system uses open source library (ZXing)[5] to produce QR code. ZXing can improve the efficiency of developing system and generate QR codes in quantities.

The special meaning of two-dimensional codes. In order to avoid collision when AGV is driving, we need to set a mark in the non-traveling area. This paper adapts the way of stacking some special two-dimensional codes in the non-driving areas. We can let the AGV stop to avoid collision because of the "Obstacle" being stored in the two-dimensional codes[6]. AGV will transmit the alert information to the control system and guide $\mathrm{AGV}$ to make corresponding obstacle avoidance behavior when the special two-dimensional code is scanned.

The planning and design of the path. The AGV described in this paper is applied in the industrial unmanned warehouse, where the movement is the driving area. The path planning of AGV under intelligent control has two forms[7], which includes path planning under static environment and under dynamic environment. They will be described in detail below.

(I) Path planning under static environment. The path planning under static environment is assumed that the robot is aware of the environment through path perception and the local area propagation algorithm is adopted. Therefore, this path is usually used in the environment when there are static known obstacles. However, a problem needs to be solved is what kind of path can be considered reasonable. This paper adapts the type which not only has the property of continuity but also can maintain the continuity in the tangential direction.

(II) Path planning under dynamic environment. The path planning under dynamic environment[8] is different from the path planning under static environment. We cannot obtain much information and 
optimal path planning due to the environmental changes. The target attraction, dynamic security and time constraint must be considered under complex environment no matter what kind of performance should be applied.

This paper uses the two-dimensional code to locate and navigate for AGV, so the different codes are posted on the driving path of AGV. The specific ideas of path planning are as follows:

The distance of two-dimensional codes should be increased when AGV is on the straight path[9]. But the distance should be narrowed down when AGV is on the turning path. It will allow AGV to update its location and drive in accordance with the specified path, so as to complete the planning path on turning path.

\section{Experimental results}

The position of two-dimensional code is shown as Fig. 4 and the results read by program is shown as Fig. 5.

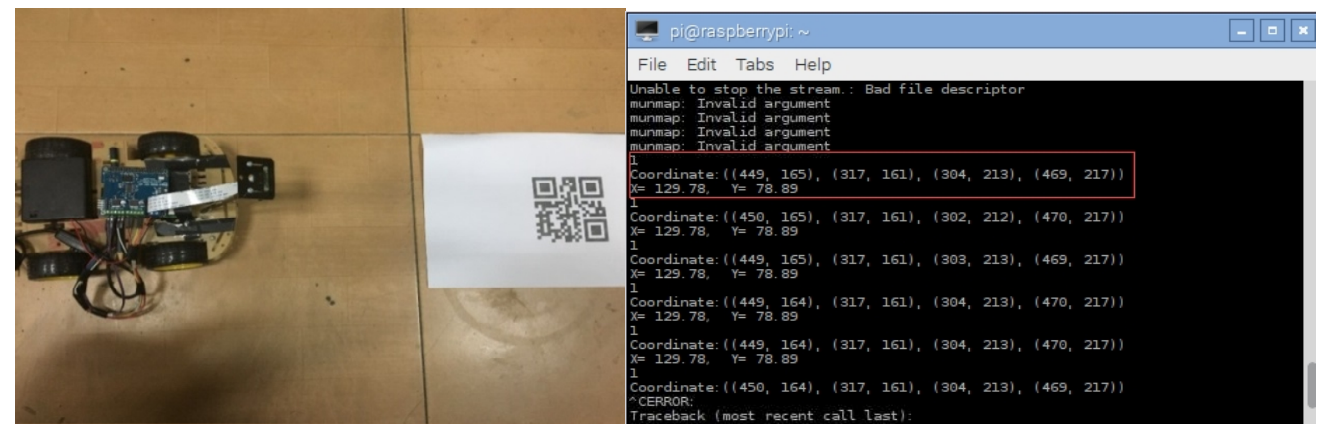

Fig. 4. Position

Fig. 5. The results read by program

We use notebook as the host machine and the communication between AGV and PC are shown as Fig. 6 and Fig. 7:

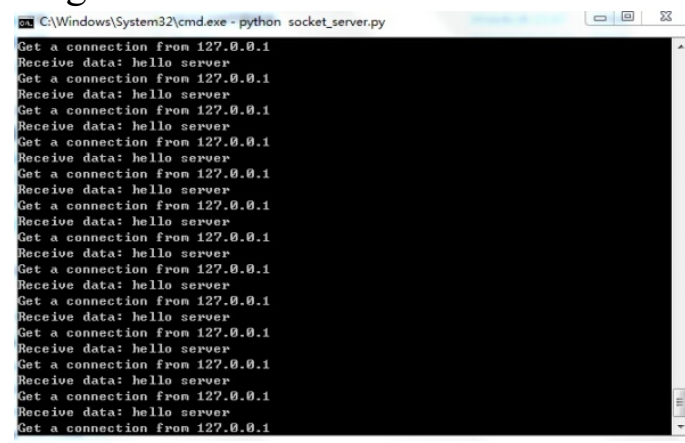

Fig. 6. The testing data

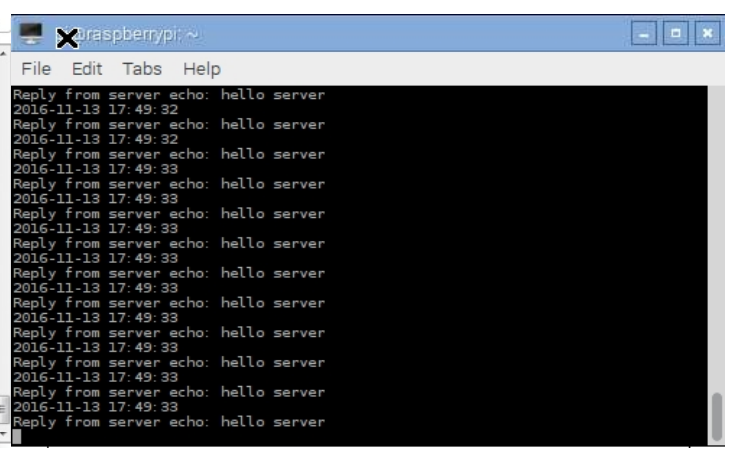

Fig. 7. The responding data from PC

The testing data include the position information of two-dimensional codes and the relative position between the codes and AGV.

The scanned real-time position information of two-dimensional codes is transmitted to the host machine, and then the PC feed the information back to AGV through the control module of it. Finally, we will realize the autonomous navigation of $\mathrm{AGV}[10]$. The figure of the movement for $\mathrm{AGV}$ is shown as Fig. 8. 


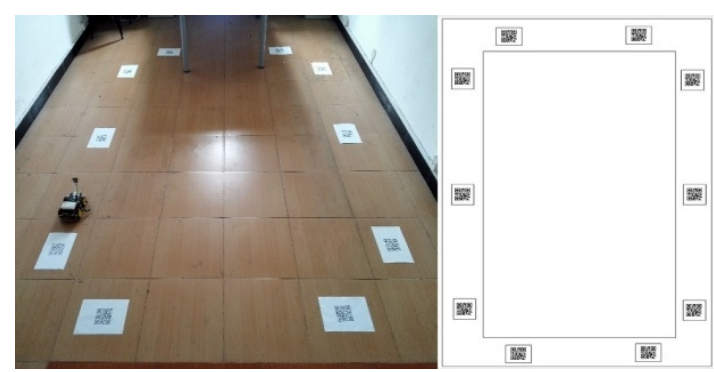

Fig. 8. The figure of the movement for AGV

\section{Conclusions}

This system solves the problem of low positioning accuracy and high costs with the help of the combination of QR 2D code and machine vision. The information of QR 2D code is scanned by ZXing. The orientation of AGV related to two-dimensional code is determined by the design principle and the scanned technology of the two-dimensional code.

The outside light has a certain influence on the scanning speed of two-dimensional code because of the system adopts the machine vision to identify the QR 2D code. Therefore, the QR 2D code[11] may be missed and it will lead to the inaccuracy of the positioning information.

\section{Acknowledgements}

This work is supported by subject of Science and Technology Commission of Shanghai Municipality in key technology development and demonstration application of the unmanned factory for industrial robot production (No. 14DZ1100700). We thank Shanghai Key Laboratory of Intelligent Manufacturing and Robotics for assistance with the automotive parts cost data.

\section{References}

[1] Rothwell C A;Zisserman A;Forsyth D A Using projective invariants for constant time library indexing in model based vision 1991.

[2] S. Willis,S. Helal, A Passive RFID Information Grid for Location and Proximity Sensing for the Blind User. University of Florida Technical Report, Oct. 2004.

[3] Jun Yang, Yan Liu, Yanrui Du. The research and application of two dimensional code[J] Applied science and technology, 2002,29(11): 11-13.

[4] Beijing Lingdong kuaipai Information Technology Co. Ltd. Snapshot two-dimensional code [EB/OL]. [2013 - 04 -12].

[5] QR wizard QRCode turn chemical [EB/OL]. [2013 -04-12].

[6] "Information capacity and versions of QR Code". Denso-Wave.

[7] Sebastián Campanario (16 September 2011). "Marketing futurista: ya se puede comprar con la cámara del celular" [Futuristic marketing: it's already possible to purchase with a cellphone's camera] (in Spanish).

[8] "QR Code Overview \& Progress of QR Code Applications" (PDF). Retrieved 26 June 2014.

[9] "Malicious Images: What's a QR Code". SANS Technology Institute. 3 August 2011. Archived from the original on 2012-07-13. Retrieved 31 August 2011.

[11] ISO/IEC 18004:2006(E) § 6.4 Data encoding; Table 3 - Number of bits in character count indicator for QR Code 2005. 